\title{
Fabrication of 2D ordered structure of self-assembled block copolymers containing gold nanoparticles
}

\author{
Chi-An Dai ${ }^{\mathrm{a}, \mathrm{b}, *}$, Yu-Lin Wu ${ }^{\mathrm{a}}$, Yi-Huan Lee ${ }^{\mathrm{b}}$, Chun-Jie Chang ${ }^{\mathrm{b}}$, Wei-Fang Su ${ }^{\mathrm{b}}$ \\ ${ }^{a}$ Department of Chemical Engineering, National Taiwan University, No. 1, Roosevelt Rd. Sec. 4, Taipei 106, Taiwan \\ ${ }^{\mathrm{b}}$ Institute of Polymer Science and Engineering, National Taiwan University, No. 1, Roosevelt Rd. Sec. 4, Taipei 106, Taiwan
}

Available online 18 January 2006

\begin{abstract}
Two-dimensional (2D) ordered structure of metal or semiconducting nanoparticles has many applications in areas of optoelectronic and biomedical technologies. Traditional top-down patterning methods like photolithography and e-beam lithography has shown to be time-consuming and expensive processes. In this study, a bottom-up method to fabricate an ordered nanodot structure with entirely wetchemical procedures is proposed by using an amphiphilic block copolymer self-assembled into well defined two/three-dimensional structures with gold nanoparticles evenly distributed in the hydrophilic domain of the copolymer. The nanodot array of micelle monolayer containing Au salts on substrates can be made by a simple dip coating process. The block copolymer micelles containing gold salts on substrates were used as nanoscale reactors for the in-situ synthesis of metal nanoparticles by chemical treatment of the film sample with hydrazine vapor. AFM was used to evaluate the uniformity of nanodots coated on a silicon wafer before and after hydrazine reduction. Transmission electron microscopy measurement showed that a monofilm of gold salts was reduced to one or a couple of gold nanoparticles inside the core volume of PVP domain by nucleation and growth mechanism upon hydrazine vapor exposure. For an exposure time longer than $5 \mathrm{~min}$, aggregation of nanodots and disruption of $2 \mathrm{D}$ ordered structure were resulted due to the swelling of PVP core by hydrazine.
\end{abstract}

(C) 2005 Published by Elsevier B.V.

PACS: 73.63.Kv; 81.07.Bc

Keywords: A1. Nanodot; B1. In-situ crystal formation; B1. Surface plasmon resonance

\section{Introduction}

Metal and semiconducting nanoparticles have been a focus of intense research due to their novel physical properties greatly different from those of their bulk materials [1,2]. Therefore, they can be used as building blocks for new materials or devices. Particularly, the formation of two-dimensional (2D) ordered arrays of nanoparticles on substrates is of increasing interest because of its application in optical, electrical, and biological technologies $[3,4]$. For example, nanoscale memory units can be made by using single electron tunneling effect on

\footnotetext{
*Corresponding author. Department of Chemical Engineering, National Taiwan University, No. 1, Roosevelt Rd. Sec. 4, Taipei 106, Taiwan. Fax: + 886223623040 .

E-mail address: polymer@ntu.edu.tw (C.-A. Dai).
}

gold nanoparticles embedded in a thin insulation layer [5]. As an another example, near-field optical data storage systems with more than tens of gigabytes may be fabricated by using an array of nanoparticles covered with a spacer layer to control the near-field optical aperture [6]. In sensor science, a 2D monolayer of gold nanoparticles have been applied to a metal substrate to enhance surface plasmon resonance effect for detecting the absorption of proteins or biological molecules [7,8].

A considerable number of methods have been developed to fabricate 2D array of nanoparticles on substrates [2]. Conventional vacuum deposition techniques, e.g. evaporation [9] and sputtering [10], can be used to fabricate a 2D array of nanoparticles. One of the more efficient methodologies for production of the 2D array of nanoparticles involves the utilization of self-assembling materials, which have an ability to generate spatially regular structures as a 
result of minimizing their thermodynamic energy [11-14]. Recently, Möller and coworkers [15,16] successfully prepared uniform gold nanoparticles and arranged them spatially in 2D arrays by using PS-PVP diblock copolymer micelles. They also studied the association behavior and stability of the micelles containing gold nanoparticles. When an excess of hydrazine was not removed after reduction of tetrachloroauric acid, which was used for a gold nanoparticle precursor, the micelles started to coagulate immediately because $\mathrm{N}_{2} \mathrm{H}_{4}$ can swell the core volume of the micelles.

In the process of making a $2 \mathrm{D}$ ordered array of nanoparticles on substrates, it is often found that one of the fabrication steps in the process may require vacuum technique, which potentially is costly and complicated. For example, the method developed by Möller and coworkers $[15,16]$ involved a vacuum technique of oxygen plasma treatment to reduce gold ions to gold nanoparticles and at the same time to remove the overcoated polymer layer.

Since the block copolymer micelles containing metal salts possess a spherical structure wherein the hydrophilic $2 \mathrm{VP}$ regions holding the metal ions may be used as 'nanoscale reactors' for the in situ synthesis of metal nanoparticles by chemical treatment of the ions [17]. The in-situ synthesis of nanoparticles is therefore very useful since the ordered structure of nanoparticles can be preserved after metal reduction.

In this paper, we report fabrication of the $2 \mathrm{D}$ ordered array of nanopartciles embedded in a structure-directing polymer layer on a substrate, using dip coating, the substrate with a solution of polystyrene-b-poly (2-vinyl pyridine) micelles containing gold salt. First, a monolayer of the copolymer containing gold salts was deposited on a silicon wafer or glass substrate. Second, the immobilized gold salts are reduced by hydrazine vapor resulting in the formation of gold nanoparticles in situ in the core volume of the micelle, thus obviating the need for using additional vacuum techniques. Third, the size of gold nanoparticles and the spacing between them can be adjusted by the molecular weight of the block copolymer and the relative amount of gold ion added in the micellar solution.

\section{Experimental procedure}

\subsection{Materials}

A series of poly(styrene- $b$-2-vinylpyridine) (PS-PVP) block copolymers of different molecular weight were polymerized by anionic polymerization. Styrene and 2-vinylpyridine were purchased from Aldrich Chemicals. Both of them were dried using $\mathrm{CaH}_{2}$ and distilled under a vacuum. 2-vinylpyridine was further purified using triethylaluminum to remove water and alcohols. The 2-vinylpyridine mixture was then purified using a repeated freeze-thaw technique followed by distillation under vacuum. sec-Butyllithium was used to first initiate styrene to form polystyrene, and its molecular weight was measured by gel permeation chromatography. Then, 2-vinylpyridine monomer was added and allowed to polymerize. The result of the molecular weight characterization of the copolymers is listed in Table 1. The polydispersities of the diblock copolymer are less than 1.1.

\subsection{Chemicals}

Hydrogen tetrachloroaurate(III), trihydrate $\left(\mathrm{HAuCl}_{4} \cdot 3\right.$ $\left.\mathrm{H}_{2} \mathrm{O}\right)$, and hydrazine hydrate $\left(\mathrm{N}_{2} \mathrm{H}_{4} x \mathrm{H}_{2} \mathrm{O}\right.$ with $64 \mathrm{wt} \%$ of hydrazine) were obtained from Aldrich Chemicals and used as received. In this study, hydrazine is used as a gas phase reducing agent for the formation of gold nanoparticles. At the room temperature, pure hydrazine is a liquid with a vapor pressure of 14.4 torr.

\subsection{Sample preparation}

The procedure for preparing PS-PVP micellar solution containing Au salts follows the method developed by Spatz et al. [15]. Therefore, a brief procedure for sample preparation is shown as below. A $0.5 \mathrm{wt} \%$ of PS-PVP block copolymer solution was made by stirring the block copolymer and toluene for at least $6 \mathrm{~h}$. Since each tetrachloroauric acid molecule form one complex with one 2-vinylpyridine unit at equilibrium, a suitable amount of $\mathrm{HAuCl}_{4}$ was added and stirred in the above micellar solution for different gold salt loading. The molar ratio between $\mathrm{HAuCl}_{4}$ and 2-vinylpyridine in copolymer is designated as $L$. After stirring for at least $24 \mathrm{~h}$, the PS-PVP $/ \mathrm{HAuCl}_{4}$ micellar solution was prepared. Silicon wafers of $1.5 \mathrm{~cm} \times 1.5 \mathrm{~cm}$ in size and carbon-coated transmission electron microscopy (TEM) copper grids were used as a substrate for dip coating. A commercial machine (KSV company, model number: LM) was used for dip coating. The substrates were mounted on sample holder, dipped into the micellar solution, immersed for $20 \mathrm{~min}$, and pulled out of the solution with a velocity of $10 \mathrm{~mm} / \mathrm{min}$. The substrates coated with a monolayer of gold salts were subject to reduction by exposure to $\mathrm{N}_{2} \mathrm{H}_{4}$ vapor for varying times.

\subsection{Transmission electron microscopy}

To prepare samples for TEM observation, carboncoated copper grids were used as substrates for dip coating micelle containing Au ion. The copper grid was dipped into

Table 1

Characteristics of the PS-PVP diblock copolymers used for this study

\begin{tabular}{lll}
\hline Code & $\begin{array}{l}\text { Mn for PS and PVP } \\
\text { block }\end{array}$ & Polydispersity index \\
\hline $\mathrm{PS}_{57 \mathrm{k}}-\mathrm{PVP}_{57 \mathrm{k}}$ & $57000-57000$ & 1.08 \\
$\mathrm{PS}_{81 \mathrm{k}}-\mathrm{PVP}_{14 \mathrm{k}}$ & $81000-14200$ & 1.07 \\
$\mathrm{PS}_{29 \mathrm{k}}-\mathrm{PVP}_{9 \mathrm{k}}$ & $29000-9000$ & 1.09 \\
\hline
\end{tabular}


micellar solution for $20 \mathrm{~min}$ followed by pulling out the copper grid with a velocity of $1 \mathrm{~mm} / \mathrm{min}$. The covered copper grids were dried by exposure to air. TEM was performed on a JEOL $1230 \mathrm{EX}$ operating at $120 \mathrm{kV}$ with Gatan dual vision CCD camera.

\subsection{Scanning force microscopy (SFM)}

A Digital Instruments SFM MultiMode ${ }^{\mathrm{TM}}$ was used in the tapping mode. In tapping mode, the stylus oscillates, touching the sample only at the end of its downward movement. The oscillation frequency for tapping mode was set to approximately $320 \mathrm{kHz}$ with $\mathrm{Si}$ cantilever which had a spring constant of about $42 \mathrm{~N} / \mathrm{m}$. The set point in the AFM control program was adjusted to change the contact force between tip and surface in order to detect the existence of gold particles inside micelle.

\section{6. $U V-V I S$ spectroscopic analysis of $P S-P V P / A U$ thick films}

The optical properties of the PS-PVP film sample containing gold nanoparticle were monitored on a thermo spectronic diode array spectrophotometer (model Helios Gamma) operated at a resolution of $2 \mathrm{~nm}$. A thick sample $(\sim 100 \mathrm{~nm}$ in thickness) of PS-PVP/Au nanoparticles was prepared for UV-VIS spectroscopic measurement by spin coating PS-PVP/HAuCl${ }_{4}$ solutions onto a quartz substrate. The reduction of $\mathrm{Au}$ ions to $\mathrm{Au}$ nanoparticles is conducted by exposing the substrate with $\mathrm{N}_{2} \mathrm{H}_{4}$ vapor.

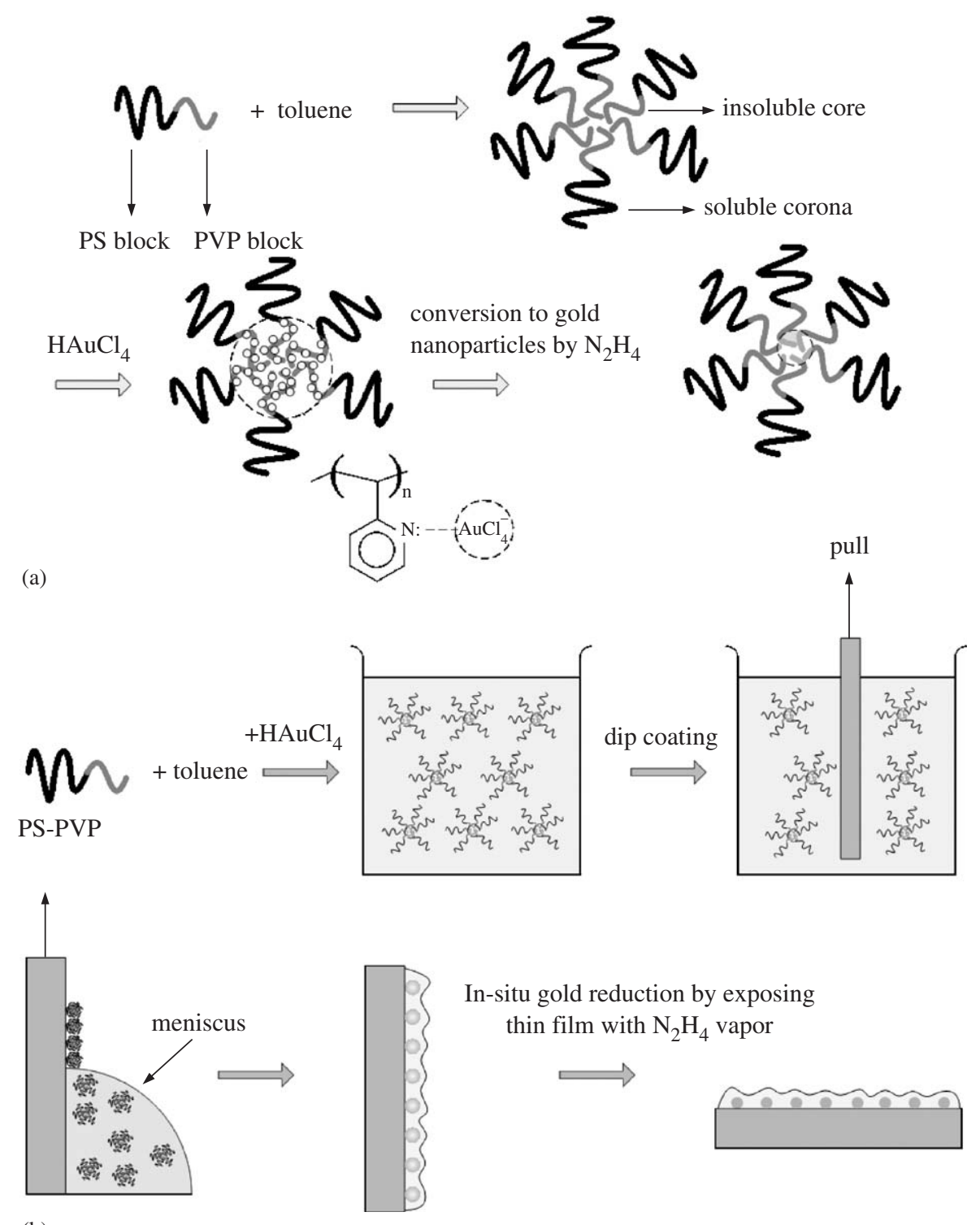

(b)

Fig. 1. (a) Schematic for the formation of gold nanoparticles inside an inversed PS-PVP micelle by $\mathrm{N}_{2} \mathrm{H}_{4}$ vapor reduction method. (b) Schematic for the formation of $2 \mathrm{D}$ ordered array of gold nanoparticles embedded in an insulating layer by in-situ $\mathrm{N}_{2} \mathrm{H}_{4}$ vapor reduction method. 


\section{Results and discussion}

The schematic for the formation of PS-PVP inverse micelle containing Au salts is shown in Fig. 1. Since PVP is not soluble but PS is soluble in toluene, an inverse micellar solution is formed when PS-PVP block copolymer is added in toluene. As $\mathrm{HAuCl}_{4}$ solid is added in the block copolymer solution, $\mathrm{AuCl}_{4}^{-}$ion forms a complex with pyridine unit. Each $\mathrm{AuCl}_{4}^{-}$ion can form one complex with one pyridine unit at equilibrium [18]. The procedure for making $2 \mathrm{D}$ ordered array of gold nanoparticles embedded in thin polymer coating is shown in Fig. 2. To prepare the thin film, a silicon substrate or a carbon-coated copper grid was dipped into the micellar solution and pulled out at a constant velocity. After drying, the films with gold salts were in-situ reduced to gold nanoparticles by direct exposure of the film with hydrazine vapor for different amount of time. The effect of exposure time of hydrazine vapor on the array structure of gold nanoparticles will be examined using AFM and TEM and discussed later in this section. In this study, different molecular weight of PS-PVP with different amount of gold salt loading inside
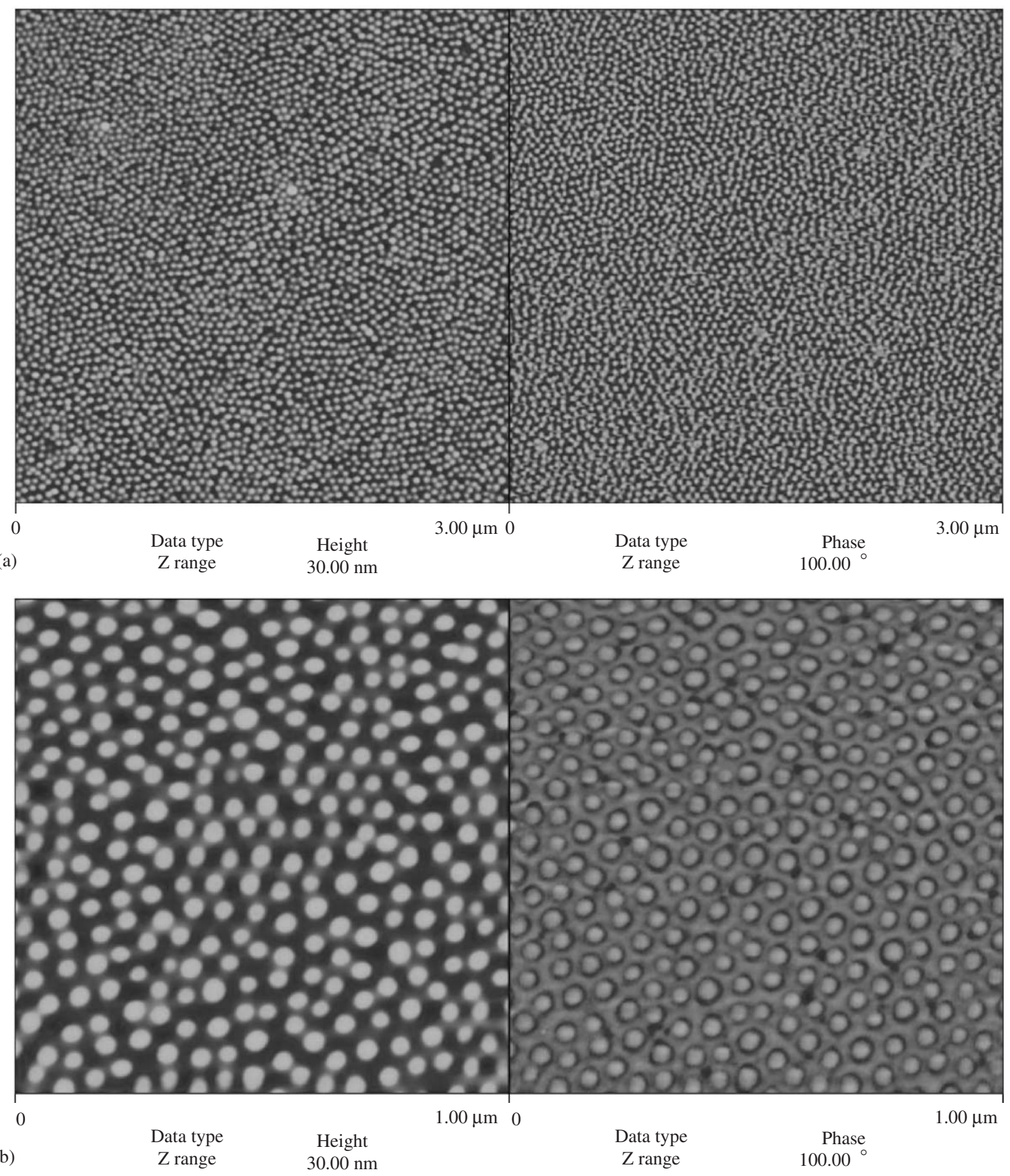

Fig. 2. The two-dimensional ordered array of $\mathrm{PS}_{57 \mathrm{k}}-\mathrm{PVP}_{57 \mathrm{k}}$ micelle containing gold salt with molar ratio $\mathrm{HAuCl} 4 / \mathrm{VP}$ of $L=0.3$ on a silicon substrate. Two different scanned sizes are shown in the above figure to show local and long-range order. The scanned size is (a) $1 \mathrm{um} \times 1 \mathrm{um}$ and (b) $5 \mathrm{um} \times 5 \mathrm{um}$. 
PVP core are studied. The core size of the monofilm is mainly determined by the molecular weight of PVP block and the gold salt loading while the spacing between each core is mainly determined by the molecular weight of PS block.

The surface morphology of a micellar monofilm of $\mathrm{PS}_{57 \mathrm{k}}-\left[\mathrm{PVP}_{57 \mathrm{k}}-\left(\mathrm{AuCl}_{4}^{-}\right)_{L=0.3}\right]$ coated on a silicon substrate before the exposure of hydrazine vapor is shown in Fig. 2(a). For all the AFM pictures shown in this study the tapping mode height and phase images are shown, respectively in the left- and right-hand sides of the AFM images. The $2 \mathrm{D}$ ordered nanodots array with some grain boundaries can be clearly seen in both AFM height and phase images over a surface area of $3 \times 3 \mu \mathrm{m}$. An even larger area of ordered nanodot structure can be made with area of up to $500 \times 500 \mu \mathrm{m}$ by using the same sample preparation procedure. Fig. 2(b) shows the detailed nanodot structure over an area of $1 \times 1$ um. The averaged
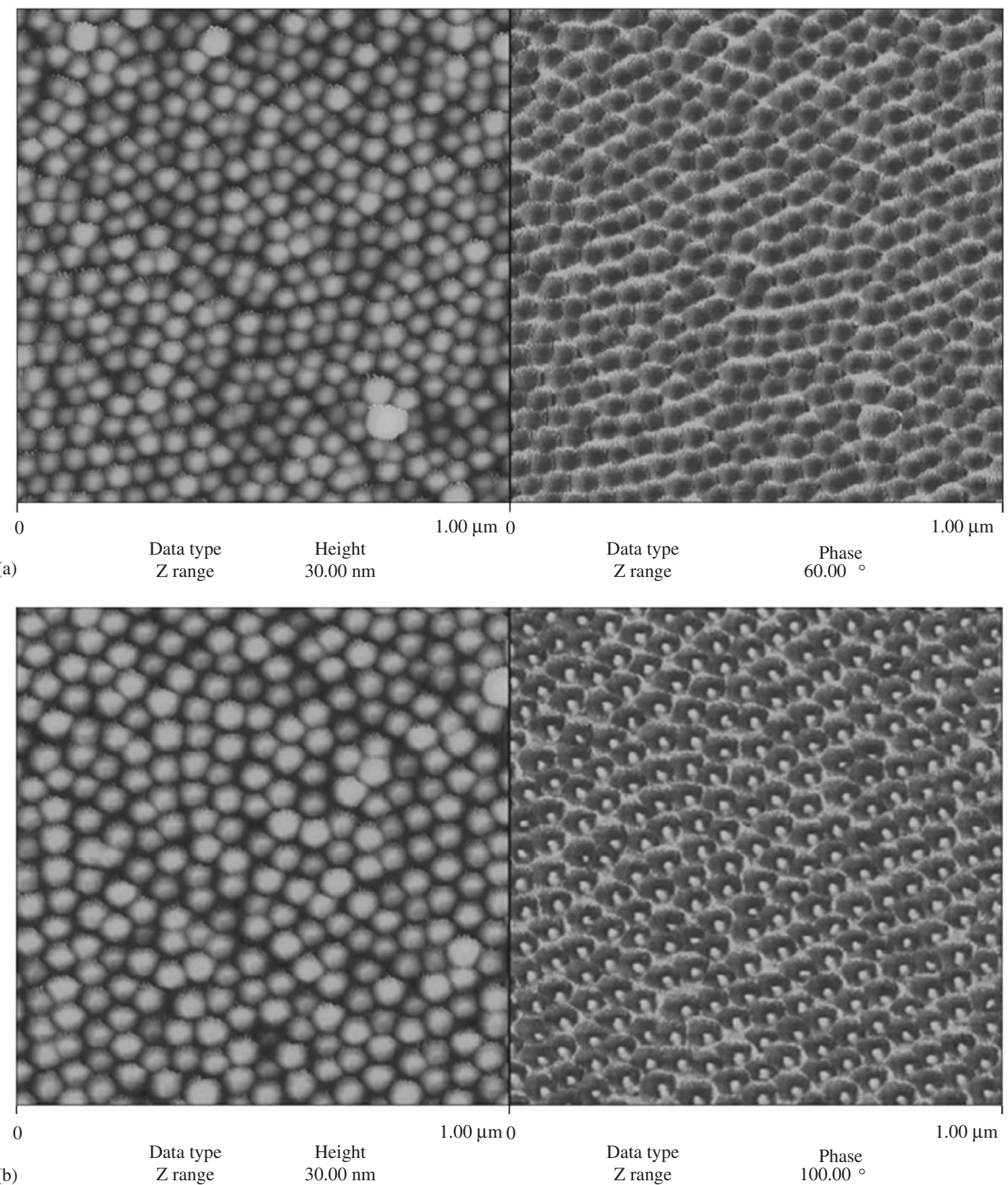

Fig. 3. The two-dimensional ordered array of $\mathrm{PS}_{57 \mathrm{k}}-\mathrm{PVP}_{57 \mathrm{k}}$ micelle containing gold nanoparticles with molar ratio $\mathrm{HAuCl} 4 / \mathrm{VP}$ of $L=0.3$ on a silicon substrate by $\mathrm{N}_{2} \mathrm{H}_{4}$ vapor reduction method. Two different tapping forces (by changing the setpoint in AFM program setting) on the film surface were used to reveal the change inside micellar film. (a) With lower tapping force, the phase diagram shows only micellar structure. (b) With higher tapping force, gold nanoparticles embedded inside the micelle layer can be clearly seen in the phase diagram on the right-hand side. 
diameter of the nanodots is roughly $40 \mathrm{~nm}$ with $\pm 5 \mathrm{~nm}$ variation and the averaged height of the nanodot is $\sim 25 \mathrm{~nm}$.

Fig. 3(a) shows that the surface morphology of the same monofilm sample after it has been exposed to hydrazine vapor for only $1 \mathrm{~min}$. After the exposure, the ordered structure of nanodots is maintained with nanodot averaged diameter of $50 \mathrm{~nm}$, slightly larger than that of the nanodots before hydrazine vapor exposure. The averaged spacing between nanodots after hydrazine exposure is decreased compared with that of nanodots before hydrazine exposure. The above results suggest that hydrazine vapor preferentially swells the PVP core of the nanodots such that the averaged diameter and the spacing between nanodots were changed accordingly.

Antonietti et al. [19] showed that PS-P4VP micelle solution containing gold salts can be reduced to a dispersion of gold colloids by the addition of $\mathrm{N}_{2} \mathrm{H}_{4}$ liquid in the micellar solution. In addition, they found that $\mathrm{Au}$ ions were evenly distributed inside the PVP core of block copolymer micelle before reduction and form $\mathrm{Au}$ nanoparticles without an ordered array structure after reduction. However, it is not known at this point whether gold salts embedded in block copolymer layer can be in situ
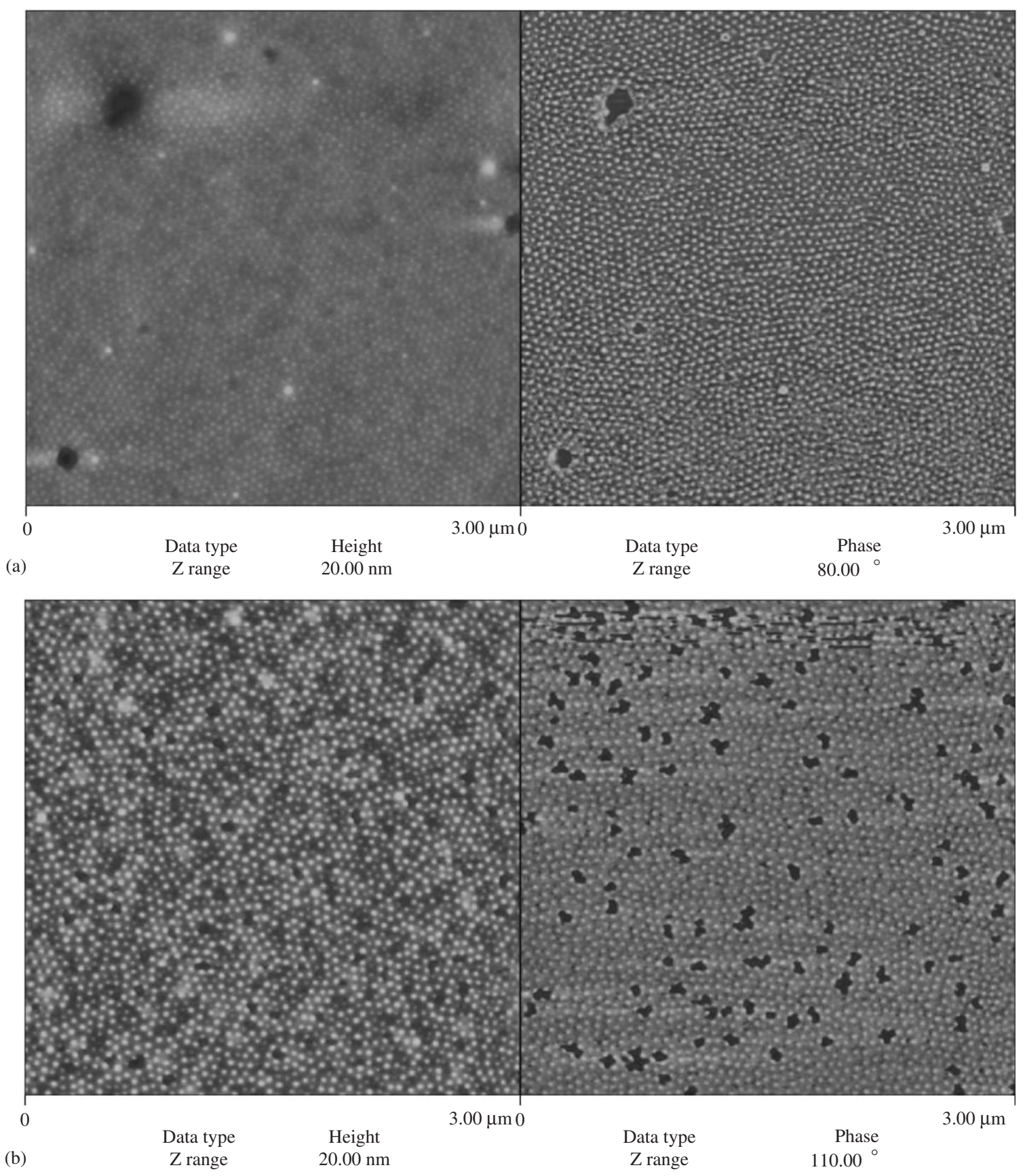

Fig. 4. The two-dimensional ordered array of $\mathrm{PS}_{81 \mathrm{k}}-\mathrm{PVP}_{14 \mathrm{k}}$ micelle containing gold salt on a silicon substrate. Two different gold salt loading were used. (a) The gold salt loading with molar ratio $\mathrm{HAuCl}_{4} / \mathrm{VP}$ of $L=0.3$. (b) The gold salt loading with molar ratio $\mathrm{HAuCl} / \mathrm{VP}_{4}$ of $L=1.0$. 
reduced to $2 \mathrm{D}$ ordered array of gold nanoparticles by brief $\mathrm{N}_{2} \mathrm{H}_{4}$ vapor exposure or not. To investigate the effect of hydrazine exposure on crystal formation using AFM, we use higher tapping force in AFM measurement to scan through the same sample which has been previously used in producing Fig. 3(a). Fig. 3(b) shows the surface morphology of the sample using higher tapping force in the AFM measurement. With higher tapping force, there appears to be small nanoparticles embedded inside the micelle layer in the phase diagram of the AFM measurement. Note that before hydrazine exposure, similar morphology cannot be obtained by using higher tapping force in the AFM measurement. Therefore, the appearance of nanoparticles inside each micelle layer indicates the existence of in-situ crystal formation of gold nanoparticles by exposing monofilm with $\mathrm{N}_{2} \mathrm{H}_{4}$ vapor.

The effects of molecular weight of PS-PVP and gold salt loading on the morphology of the monofilm were investigated using $\mathrm{PS}_{81 \mathrm{k}}-\mathrm{PVP}_{14 \mathrm{k}}$ block copolymer. Fig. 4(a) and (b) depict samples that were prepared from a toluene solution of $\mathrm{PS}_{81 \mathrm{k}}-\left[\mathrm{PVP}_{14 \mathrm{k}}-\left(\mathrm{AuCl}_{4}^{-}\right)\right]$with molar ratio $L=0.5$ and 1.0 , respectively. By comparing the surface morphology of $\mathrm{PS}_{57 \mathrm{k}}-\left[\mathrm{PVP}_{57 \mathrm{k}}-\left(\mathrm{AuCl}_{4}^{-}\right)_{L=0.3}\right]$ shown in Fig. 2(a), it is clear that the nanodots made from $\mathrm{PS}_{81 \mathrm{k}}-\left[\mathrm{PVP}_{14 \mathrm{k}}-\left(\mathrm{AuCl}_{4}^{-}\right)_{L=0.5}\right]$ is smaller and the spacing between nanodots is larger which is consistent with their molecular weight of PS and PVP block and gold salt loading. Since the PS block in $\mathrm{PS}_{81 \mathrm{k}}-\mathrm{PVP}_{14 \mathrm{k}}$ is much longer, its space-filling ability on silicon substrates is affected. Therefore, it can be seen from Fig. 4(a) that there is some area which is not covered by the copolymer hybrid film. With an increase in the gold salt loading $(L=1.0)$, such space-filling ability is greatly affected and many defects in which there is no copolymer coating were resulted as shown in Fig. 4(b)

Förster and Antonietti [17] demonstrated with their TEM measurements that depending on the activity of reducing agents used, the morphology of gold nanoparticles changes from a large number of small gold nanoparticles within one microcompartment to only one large gold nanoparticles within the PVP core volume. In our current study, in order to verify that there are gold nanoparticles still embedded in the micelles TEM measurement of the film sample is necessary. Fig. 5 shows $\mathrm{PS}_{57 \mathrm{k}}-\left[\mathrm{PVP}_{57 \mathrm{k}}-\left(\mathrm{AuCl}_{4}^{-}\right)_{L=0.3}\right]$ and $\mathrm{PS}_{81 \mathrm{k}}-\left[\mathrm{PVP}_{14 \mathrm{k}}-\left(\mathrm{AuCl}_{4}^{-}\right)_{L=0.5}\right]$ hybrid films before and after hydrazine exposure. Although the exposure time is short, it is clearly seen from Fig. 5(b) and (d) that gold salts have been reduced to gold nanopartilces embedded in PVP core of the micelle.

The effect of micellar solvent on the 2D nanodot structure is also studied. In this aspect, tetrahydrofuran (THF) instead of toluene is used as a solvent for making the micellar solution. Although PS, PVP and $\mathrm{HAuCl}_{4}$ are all soluble in THF, a micellar solution can be made since $\mathrm{AuCl}_{4}^{-}$ions form a better coordination with soft PVP block than with harder THF molecules [20]. Fig. 6(a) and (b) show the surface morphology of $\mathrm{PS}_{29 \mathrm{k}}-\mathrm{PVP}_{9 \mathrm{k}}-\left(\mathrm{AuCl}_{4}^{-}\right.$ )$_{L=0.5}$ ] monofilm coated from a THF micellar solution on a silicon substrate before and after, respectively hydrazine vapor exposure. Note that although the molecular weight of the PVP block is only 9000, much smaller than that of PVP block in $\mathrm{PS}_{57 \mathrm{k}}-\mathrm{PVP}_{57 \mathrm{k}}$ and $\mathrm{PS}_{81 \mathrm{k}}-\mathrm{PVP}_{14 \mathrm{k}}$ used in the
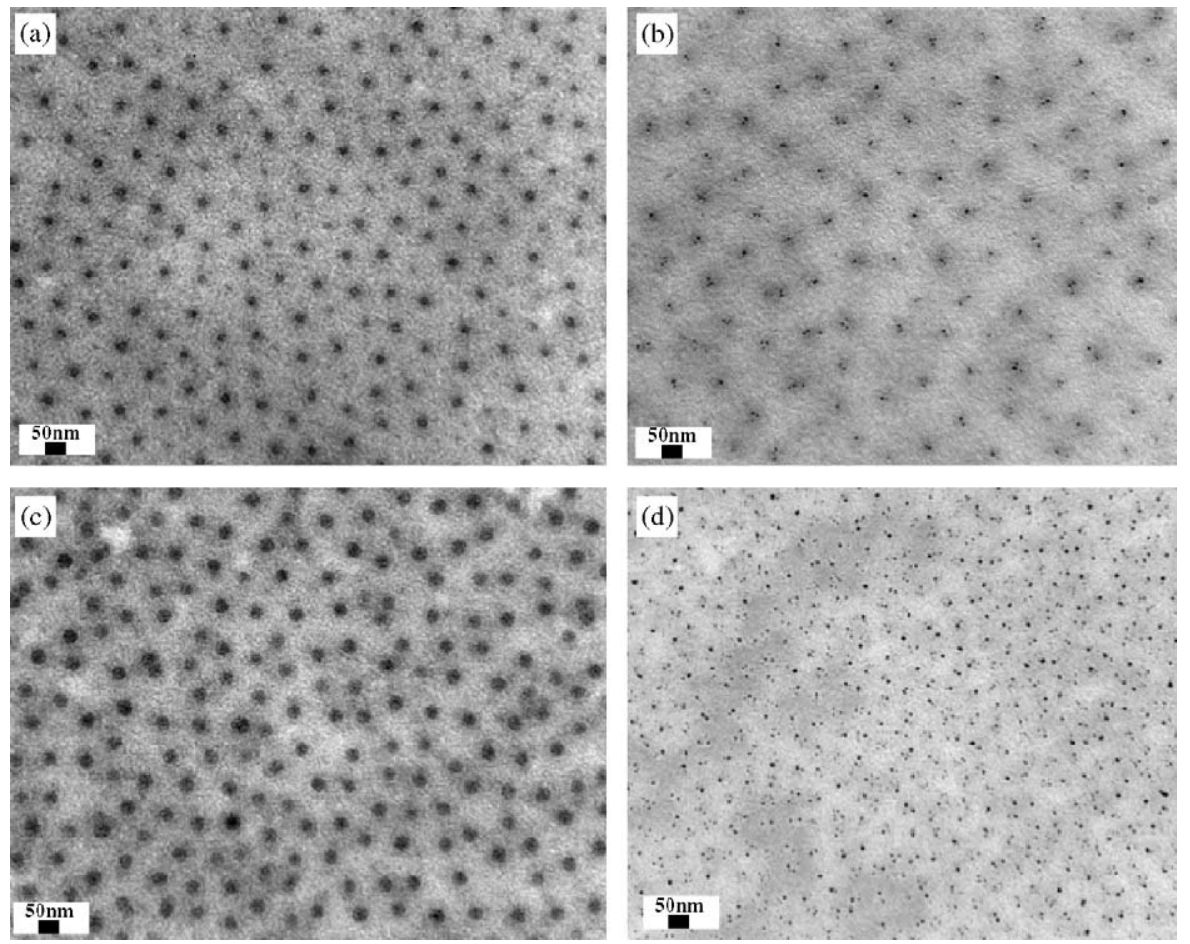

Fig. 5. TEM micrographs of two-dimensional ordered array of $\mathrm{PS}_{81 \mathrm{k}}-\mathrm{PVP}_{14 \mathrm{k}} / \mathrm{HAuCl}_{4}$ hybrid film (a) before $\mathrm{N}_{2} \mathrm{H}_{4}$ vapor reduction (b) after $\mathrm{N}_{2} \mathrm{H}_{4}$ vapor reduction. For $\mathrm{PS}_{57 \mathrm{k}}-\mathrm{PVP}_{57 \mathrm{k}} / \mathrm{HAuCl}$ hybrid film, TEM shows the array structure(c) before $\mathrm{N}_{2} \mathrm{H}_{4}$ vapor reduction (d) after $\mathrm{N}_{2} \mathrm{H}_{4}$ vapor reduction. 

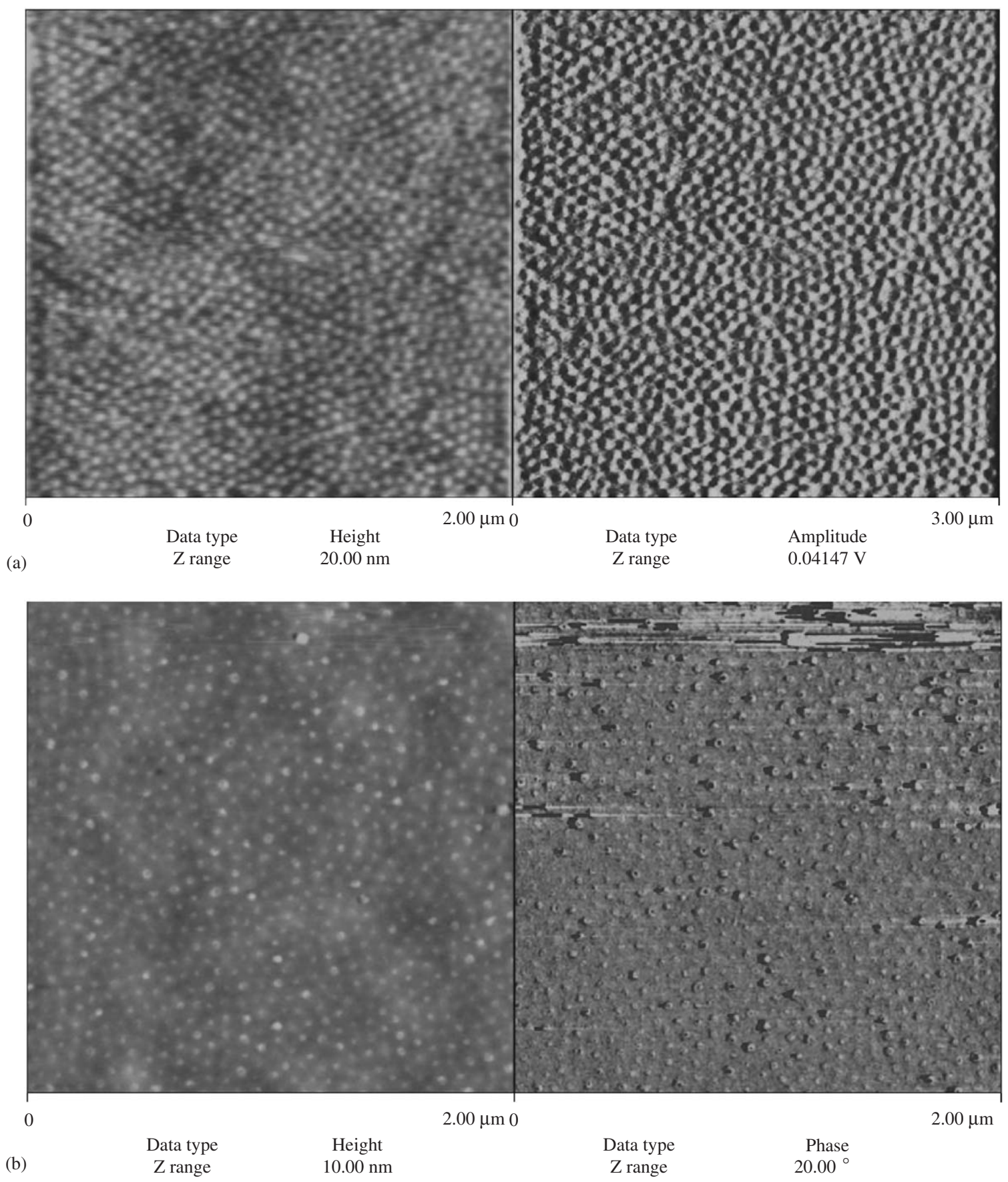

Fig. 6. The effect of micellar solvent on the nanodot structure (a) before and (b) after hydrazine reduction for $\mathrm{PS}_{29 \mathrm{k}}-\mathrm{PVP}_{9 \mathrm{k}} / \mathrm{HAuCl}_{4}(L=0.5)$ hybrid film. Instead of using toluene as a solvent, nanodot structure was made by using THF.

previous section, the nanodots formed from THF with averaged diameter of $50 \mathrm{~nm}$ is bigger than those formed from $\mathrm{PS}_{57 \mathrm{k}}-\mathrm{PVP}_{57 \mathrm{k}}$ and $\mathrm{PS}_{81 \mathrm{k}}-\mathrm{PVP}_{14 \mathrm{k}}$ toluene solutions. The expanded size effect can be attributed to the swelling effect of the PVP core by THF solvent. After exposure of hydrazine vapor for just $1 \mathrm{~min}$, there is a distinct change in the nanodot morphology. Nanodots shrink from 50 to $40 \mathrm{~nm}$ in diameter and from 20 to $10 \mathrm{~nm}$ in height as shown in Fig. 6(b). Note that such shrinkage in core size was not observed in samples coated from toluene solution of PS-PVP.

To demonstrate the unique optical property of an ordered array of gold nanoparticles, we prepared a thick film ( $\sim 100 \mathrm{~nm}$ in thickness) coated with PS-PVP/Au hybrid film made from in-situ reduction of gold salts to gold nanoparticles embedded in the thick film. We performed the UV-VIS absorption measurement on a thick film with thickness generally greater than $100 \mathrm{~nm}$ since the optical 


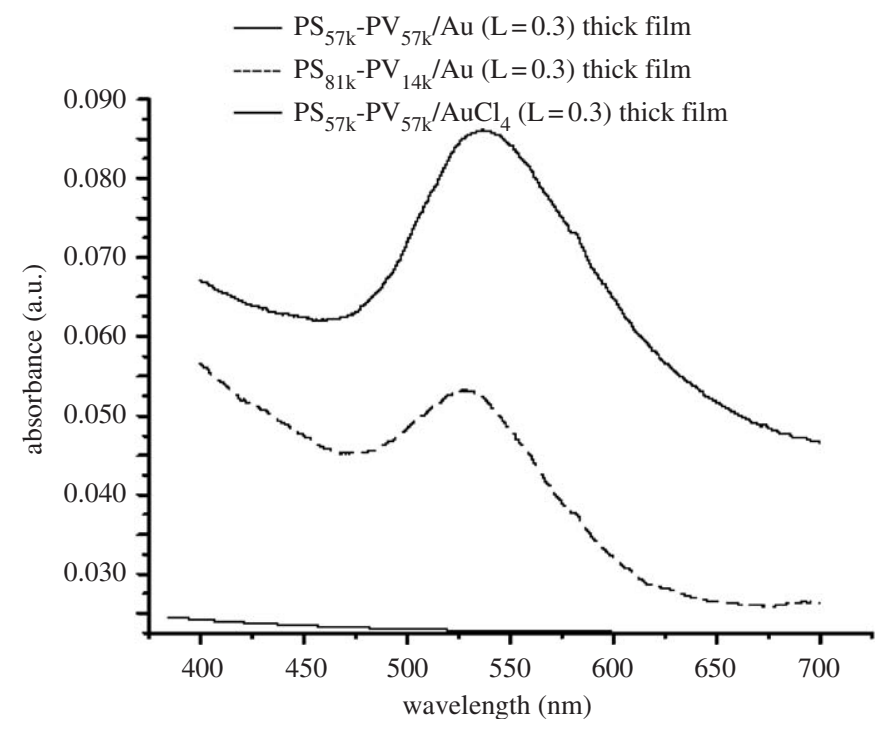

Fig. 7. UV-VIS absorption of a PS-PVP/HAuCl 4 thick film before and after hydrazine exposure. After hydrazine exposure, a characteristic surface plasmon resonance absorption peak $(\sim 560 \mathrm{~nm})$ from gold nanoparticles is clearly seen.

absorption of a thin monofilm of nanodots containing gold nanoparticles is usually not detectable using conventional UV-VIS measurement. The UV-VIS absorption spectrum of a PS-PVP $/ \mathrm{HAuCl}_{4}$ thick film before and after hydrazine exposure is shown in Fig. 7. Before gold nanoparticles was reduced, there is almost no absorption in the range of $400-700 \mathrm{~nm}$ for PS-PVP/HAuCl${ }_{4}$ hybrid film. After exposing to hydrazine vapor, hybrid films containing $\mathrm{PS}_{57 \mathrm{k}}-\mathrm{PVP}_{57 \mathrm{k}} / \mathrm{Au}$ and $\mathrm{PS}_{81 \mathrm{k}}-\mathrm{PVP}_{14 \mathrm{k}} / \mathrm{Au}$ show a characteristic surface plasmon absorption peak in the UV-VIS spectra. The UV-VIS measurements also demonstrate the effectiveness of the method in reducing gold salt and potential prove the concept of using self-assembling block copolymer for the fabrication of $2 \mathrm{D}$ ordered array of nanoparticles embedded in block copolymer micelle. The existence of the optical characteristics of $2 \mathrm{D}$ ordered monolayer of $\mathrm{Au}$ nanoparticles can be potentially used in many optoelectronic application.

\section{Conclusion}

In this study, we fabricate an ordered nanodot structure with entirely wet-chemical procedures using an amphiphilic block copolymer self-assembled into well defined 2D structures with gold nanoparticles evenly distributed in the hydrophilic domain of the copolymer. The nanodot array of micelle monolayer containing $\mathrm{Au}$ salts on substrates can be made by a simple dip coating process followed by in-situ synthesis of metal nanoparticles by chemical treatment of the film sample with hydrazine vapor.

AFM and TEM measurements show that an ordered array of monofilm film containing gold nanoparticles can be made from hydrazine exposure and the gold salts can be converted to gold nanoparticles inside the core volume of
PVP domain by nucleation and growth mechanism. By adjusting tapping force in the AFM measurement, gold nanoparticles embedded inside the block copolymer can be directly imaged. This result is consistent with TEM observation that gold nanoparticles form 2D ordered array inside PVP core volume upon hydrazine exposure.

AFM and TEM measurement also show that the size of gold nanoparticles and the spacing between them can be adjusted by the molecular weight of the block copolymer and the relative amount of gold ion added in the micellar solution. UV-VIS measurement shows that PS-PVP/Au thick film gives a characteristic surface plasmon resonance absorption which can potentially be used in the optoelectronic application.

\section{Acknowledgement}

This work is supported by the National Science Council of Taiwan under contract numbers NSC92-2216-E-002-015 and NSC93-2120-M-002-010 and US Air Force under contract number AFOSR-AOARD-04-4023.

\section{References}

[1] V.I. Klimov (Ed.), Semiconductor and Metal Nanocrystals-Synthesis and Electronic and Optical Properties, Marcel Dekker, Inc., New York, 2004.

[2] P.N. Prasad, Nanophotonics, Wiley, New York, 2004.

[3] G. Kastle, H.-G. Boyen, F. Weigl, G. Lengl, T. Herzog, P. Ziemann, S. Riethmuller, O. Mayer, C. Hartmann, J.P. Spatz, M. Moller, M. Ozawa, F. Banhart, M.G. Garnier, P. Oelhafen, Adv. Funct. Mater. 13 (2003) 853.

[4] J.H. Fendler (Ed.), Nanoparticles and Nanostructured Films, WileyVCH, Weinheim, 1998.

[5] S.-T. Yau, P. Mulvaney, W. Xu, G.M. Spinks, Phys. Rev. B 57 (1998) R15124.

[6] D.P. Tsai, W.C. Lin, Appl. Phys. Lett. 77 (2000) 1413.

[7] E. Hutter, S. Cha, J.-F. Liu, J. Park, J. Yi, J.H. Fendler, D.J. Roy, Phys. Chem. B 105 (2001) 8

[8] L.A. Lyon, M.D. Musick, P.C. Smith, B.D. Reiss, D.J. Pena, M.J. Natan, Sensors Actuators B 54 (1999) 118.

[9] T.L. Morkved, P. Wiltzius, H.M. Jaeger, D.G. Grier, T.A. Witten, Appl. Phys. Lett. 64 (1994) 422.

[10] R. Chandra, P. Taneja, J. John, P. Ayyub, G.K. Dey, S.K Kulshreshtha, Nanostruct. Mater. 11 (1999) 1171.

[11] R.G. Freeman, C. Grabar, K.J. Allison, R.M. Bright, J.A. Davis, A.P. Guthrie, M.B. Hommer, M.A. Jackson, P.C. Smith, D.G. Walter, M.J. Natan, Science 267 (1995) 1629.

[12] C.S. Chen, M. Krksich, S. Hang, G.M. Whitesides, D.E. Ingber, Science 276 (1997) 1425.

[13] S. Sun, C.B. Murray, D. Weller, J. Folks, A. Moser, Science 287 (2000) 1989.

[14] T. Hayakawa, Y. Usui, S. Bharathi, M. Nogami, Adv. Mater. 16 (2004) 1408.

[15] J.P. Spatz, S. Mossmer, C. Hartmann, M. Moller, Langmuir 16 (2000) 407.

[16] S. Mossmer, J.P. Spatz, M. Moller, T. Aberle, J. Schmidt, W. Burchard, Macromolecules 33 (2000) 4791.

[17] S. Forster, M. Antonietti, Adv. Mater. 10 (1998) 195.

[18] J.P. Spatz, S.S. Sheiko, M. Moller, Macromolecules 29 (1996) 3220.

[19] M. Antonietti, A. Thunemann, E. Wenz, Colloid Polym. Sci. 174 (1996) 795.

[20] E. Wenz, Ph.D. Thesis, Freie Universitat Berlin, 1996. 